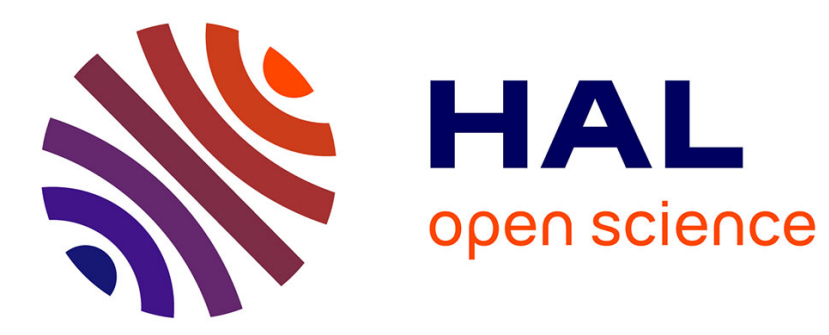

\title{
A Study of Mobility Support in Wearable Health Monitoring Systems: Design Framework
}

\author{
Amine Boulemtafes, Abderrezak Rachedi, Nadjib Badache
}

\section{To cite this version:}

Amine Boulemtafes, Abderrezak Rachedi, Nadjib Badache. A Study of Mobility Support in Wearable Health Monitoring Systems: Design Framework. 12th ACS/IEEE International Conference on Computer Systems and Applications, Nov 2015, Marrakech, Morocco. hal-01260539

\section{HAL Id: hal-01260539 \\ https://hal.science/hal-01260539}

Submitted on 26 Jan 2016

HAL is a multi-disciplinary open access archive for the deposit and dissemination of scientific research documents, whether they are published or not. The documents may come from teaching and research institutions in France or abroad, or from public or private research centers.
L'archive ouverte pluridisciplinaire HAL, est destinée au dépôt et à la diffusion de documents scientifiques de niveau recherche, publiés ou non, émanant des établissements d'enseignement et de recherche français ou étrangers, des laboratoires publics ou privés. 


\section{A Study of Mobility Support in Wearable Health Monitoring Systems: Design Framework}

\author{
Amine Boulemtafes \\ CERIST, Algiers, Algeria \\ University M. A. Béjaia, Algeria \\ aboulemtafes@cerist.dz
}

\author{
Abderrezak Rachedi \\ University Paris-Est (UPEM) \\ Computer Science Lab. (LIGM) \\ rachedi@u-pem.fr
}

\author{
Nadjib Badache \\ CERIST, Algiers, Algeria \\ USTHB, Algiers, Algeria \\ badache@cerist.dz
}

\begin{abstract}
The aim of this work is to investigate main techniques and technologies enabling user's mobility in wearable health monitoring systems. For this, design requirements for key enabling mechanisms are pointed out, and a number of conceptual and technological recommendations are presented. The whole is schematized and presented into the form of a design framework covering design layers and taking in consideration patient context constraints. This work aspires to bring a further contribution for the conception and possibly the evaluation of health monitoring systems with full support of mobility offering freedom to users while enhancing their life quality
\end{abstract}

Keywords-Health monitoring, wearable devices, wireless body area network (WBAN), e-Health, m-health, ageing, mobility

\section{INTRODUCTION}

Continuous health monitoring especially for elderly and impotent patients becomes more and more necessary. The ageing of the world population added to the increase of health costs have been the principal motivations for the design of such monitoring systems [1]. The development of a special type of wireless sensor Networks (WSNs) called Wireless Body Area Network (WBAN), was directly triggered by the unsuitability of WSN for monitoring human body [2, 3]. Such systems called wearable health monitoring systems (WHMS) based on wearable sensors, in addition to allow the decrease of health costs, are also made in order to enhance quality of life of patients becoming more independent. For this, it seems important that HMS need to support non restricted mobility for monitored users as well as health professionals which enables an anywhere and anytime monitoring, allowing an instant detection of abnormal health cases, predicting and preventing serious and critical health problems such as falls and thus enabling immediate reaction towards the patient [4,5].

However, user's mobility implying contextual information changes such as available resources and location, needs specific requirements to be fulfill, and leads to various issues and challenges such as intermittent connectivity or wireless technologies interferences. These combining multiple research areas related to WHMS' design, require a certain number of techniques and technologies for a seamless mobility support $[5,8,9]$.

Examining the literature, a number of research studies and proposals addressing or including mobility support in HMS exist. In [44], authors proposing a framework for comparison of health monitoring systems, considered mobility of patients through wireless communications aspects since mobility is said to be maximally supported through wireless communication technologies. Authors in [11], within a survey of requirements for health monitoring systems, considered mobility support through techniques to adapt to broken routes and fluctuating transmission link quality. In [45], authors supported mobility within their proposed data collection system through cost effective communication by switching between available networks according to current region and using buffering with postponed transmissions in order to handle intermittent connectivity. Other studies and research works addressed and focused on some of the key mechanisms for mobility support such as handover [9,12], coexistence handling $[13,14,15]$, intermittent connectivity handling [20] or localization [16]. However, to the best of our knowledge, none of these studies addressed mobility support from a perspective describing its design requirements and their correlation with user's context. Addressing mobility support from such perspective, aims to give a clear view of requirements and challenges, and techniques and technologies around the subject, which possibly contribute in the conception of efficient WHMS.

This work therefore highlights key means enabling full mobility support in WHMS. For this, main aspects around mobility support from techniques and technologies to surrounding contexts of the user are reviewed, discussed and correlated within our framework proposal. This, aspire to be a starting point for the conception of WHMS with full support of mobility while also attempts to provide an assessment platform helping to evaluate mobility support of monitoring systems and projects.

This paper is organized as follow: in section 2, preliminary concepts related to the design framework are presented. Section 3 details and discusses the proposal including key mechanisms and recommendations, while section 4 concludes the paper along with future perspectives.

\section{FOREWORD}

WHMS are generally designed on the basis of the typical three-tiers architecture composed from intra, inter and beyond BAN levels and whose the core idea is the use of any existing ICT-based wireless communication to achieve cooperation between these three components [2]. It is reported that using a three-tiers representation considered as a complete model is a reasonable choice for remote HMS [2]. For this, three-tiers representation will be the starting architectural point for this work. 
Besides, supporting non-restricted user mobility implies different locations around which a patient can roam such as home, hospital or public parks. Besides, systems supporting delimited area mobility, exploit specific location advantages; such advantages can also be exploited in case of non-restricted mobility. Therefore, a HMS supporting a non-restricted mobility, allowing the user to move anywhere, would ideally take at the same time advantages of different locations the patient could meet. Within this outlook, this work further addresses in-home indoor environment and its specificities, as a special location for patient, in order to take advantage of, as this specific environment can be considered as a major typical and totally customizable location.

From another hand, user mobility, being part of the patient context, could not be isolated; instead, techniques and technologies used to support it are rather in close relation with this context. Communications are particularly impacted by user mobility, more especially transmission of patient's data. Therefore, any mean for supporting mobility might be dependant of patient's data profile whether it's in term of frequency, reliability or any other profile constraints as it will be described below. Moreover, patient's location is also an important parameter on which mobility support means might be dependant in term of available communication and sensing infrastructures for example or suitable techniques adapted to the environment.

\section{DESIGN FRAMEWORK FOR MOBILITY SUPPORT}

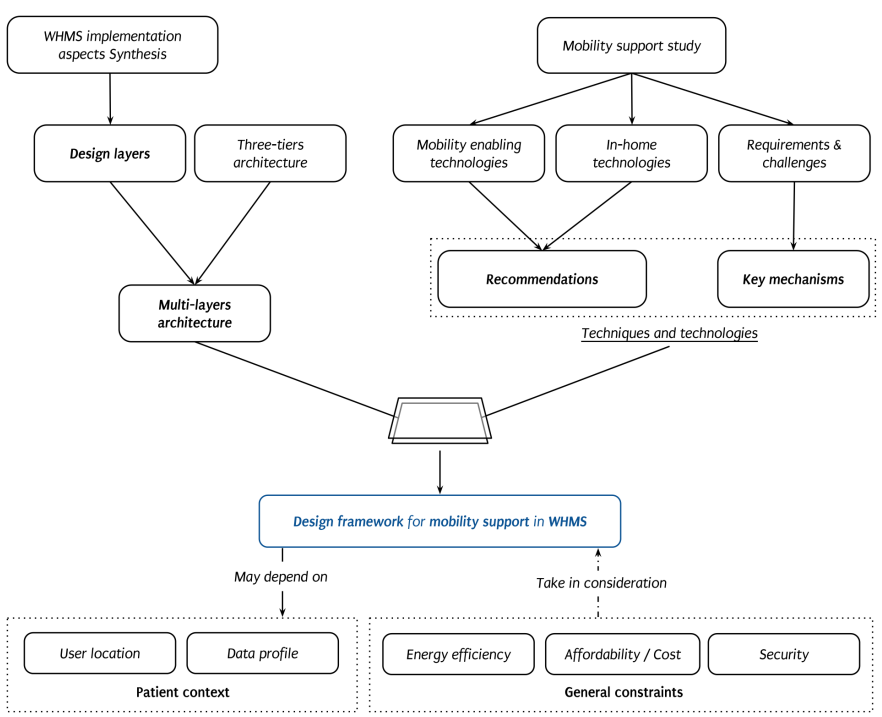

Fig. 1. Followed methodology

In order to shape our design framework proposal (see Fig. 1): (1) we introduce a multi-layers architecture based on the typical three-tiers representation [2], aiming to cover major involved WHMS design aspects. This architecture will serve as a basis for our design framework; (2) we study some of the main relevant elements of the patient context i.e. data profile and patient location, and extract different context constraints impacting mobility support means; (3) major techniques and technologies for supporting user mobility in WHMS are defined in the form of conceptual recommendations and key mechanisms' design requirements; (4) techniques and technologies for mobility support are correlated with different defined constraints then overlaid on the multi-layers architecture forming the design framework proposal.

\section{A. Multi-layers architecture}

A HMS is typically built of three building blocks namely sensing, communication and data analysis, considered as the three main functionalities enabling HMS to operate [6].

\section{Design layers}

However, main building blocks represent only the basis of design layers to be considered in patient monitoring systems. From a wider perspective, other levels complementing these should be considered in order to have a complete view of how such systems can be implemented and built. These should involve all major aspects related to the design of patient monitoring systems.

Examining the literature regarding HMS implementation and starting from main building blocks, a number of design aspects can be spotted. These are introduced here as design layers proposal as follows:

1) Hardware layer: Involves all different hardware used in the system including, such as sensor nodes and base station at the intra-BAN level, different gateways, relays and other communication hardware at the inter-BAN level or remote server(s) at beyond-BAN level

2) Communications layer: Includes standards, MAC, routing, and other upper layer protocols, and this at each level;

3) Low-level processing layer: Involves different mechanisms of signal and data raw processing ie. without information analysis and extraction including denoising techniques, compression, encoding/decoding, ...etc.

4) Semantic processing layer: Involves different processing mechanisms like data analysis techniques, data mining algorithms, ... etc. whose the aim is to extract relevant information related to patient health status or other nonmedical information such as user connectivity or location.

5) System operation layer: Involves different non-data centric mechanisms that enable system's operation including on-node or on base station procedures such as authentication algorithms, failure detection, alerts generation at intra-BAN level, or at beyond-BAN level, including procedures for authentication, failure detection and recovery, for data storage as well as other back-end components of possible applications.

6) Front-end layer: Involves different interactions between users (patient, health professionals, ...etc.) and the system including needed interactions and displaying on sensor nodes and the base station at intra-BAN level such as displaying relevant health information to the user, as well as needed interactions and displaying at beyond-BAN such as mobile or web application for monitoring users health status and setting parameters.

\section{B. Patient context: design requirements}

As user mobility is a part of the patient context, techniques and technologies for its support could not be disassociated from this context's constraints but are rather in close relation. In fact, since communications are subject to be impacted by mobility, supporting techniques and technologies might be dependent from the profile of transmitted data such as their frequency or reliability requirement. Besides, location of the patient is also an important factor on which these techniques 
and technologies might be dependent such as available communication networks or sensing infrastructures.

Therefore, three major aspects and their impact are interesting for our design framework, (1) the mobility of the patient which is the basis for constraints defining design requirements and mechanisms, and (2) the patient location, as well as (3) the profile of data to be monitored, both representing the selection indicator for customization.

From another hand, typical HMS' requirements and constraints should also be taken in consideration. Three major of these are energy efficiency for continuous long monitoring, cost of the solution or affordability for wide public access and security as it comes to sensitive information.

1) Monitored data profile: Before going through different design requirements and mechanisms used in order to support non-restricted mobility in WHMS, it is worth to explore the context of the monitored patient in term of the data to be collected, which we believe is one of the most important aspects for the customization of hardware, standards or mechanisms to be used.

Through the literature and examining patient's data profile, we propose to define a set of related constraints, namely: (1) Data collection frequency requirement $(D F)$, defining how certain data should be collected in term of time intervals of sensing $[8,18,35]$; (2) Data processing requirement (DPC), referring to the need to certain data to be processed or not (used directly) as well as the nature of the processing performed [25,37]; (3) Real-time requirement ( $R T)$, defining whether a specific monitored data should be on-time delivered and/or processed in real-time or is rather delay tolerant [6,37]; (4) Data reliability requirement (DR), referring to the need of data quality i.e. if a specific monitored data is loss and/or error-tolerant [11,36]; (5) Data prioritization requirement $(D P R)$, where some data or alerts might be more critical than others, and therefore would be more prioritary than routine data such as in emergency cases where some vital signs becomes more sensitive as well as alerts [18]; (6) Data correlation requirement (DC), leading to a need of synchronization between some data, if these are somehow associated and/or need to be combined [18,25]; (7) Data management requirement (DM), defining if monitored data settings are fixed and set only the first time or if it should be manageable such as setting thresholds, enabling/disabling the monitoring of some data ... etc. $[1,18]$.

Answering these data profile constraints according to the target patient, helps in the selection and customization of design requirements and mechanisms related to non-restricted mobility support as it will be shown below.

2) Non-restricted mobility: Mobility for WHMS can be divided into two main categories: (1) Network mobility $[38,39]$, typically where an entire WBAN ie. monitored person moves; and (2) intra-WBAN Mobility [40], where individual nodes moves due to human body movements; this, has to be typically always considered since a human body is always in movement regardless of the target application except for some rare cases of disabled persons.

Through the study of non-restricted user mobility support in WHMS which is the fundamental starting aspect of our patient context, a number of recommendations as well as a set of required mechanisms can be distinguished. Sensor network around the patient would be location-based i.e. in-home (ihBAN) and outdoor (out-BAN), while other communication networks availability would depend on the patient's location. Recommendations as well as mechanisms described in the form of a set of design requirements are therefore correlated to the data profile constraints and location-based BANs.

\section{Recommendations}

Data profile requirements are associated with recommendations illustrating their impact on these latter's parameters or squarely their need.

\section{Intra-BAN level}

- 1) Base architecture. Three-tiers architecture considered as a complete model in HMS [2].

- 2) Sensors design. Lightweight, unobtrusive and noninvasive design [17], energy-efficient as a user being mobile could not be able to recharge devices for long periods, and low-cost in order to be as publicly accessible as possible. Tamper resistant and hermetically sealed to reduce fraud possibility, while mechanisms such as electromagnetic interference filters need to be considered $[3,18]$. Sensors should also be designed in a way to detect and mitigate movements' interferences which might be dependant of data reliability in term of error tolerance. Energy efficiency constraint might depend on data frequency requirement, while affordability is in relation with data reliability and frequency.

- 3) Base station (BS). Mobile platforms are well adapted to be used as base station in HMS especially Smartphones which are more popular [19]. These are becoming pervasive computing platforms providing ubiquitous communication and services [19], they support different wireless technologies and are more and more equipped with low energy communication technologies, all this contributing in its role as gateway, emergency situation handling and energy efficiency. Smartphones are also built-in with sensors such as GPS, and offer typically quite powerful storage and processing capacities contributing in different mechanisms for the mobility support such as localization, and allow the use of algorithms with high compression ratio, contributing in cost reducing and energy saving [20,9]. The choice of the mobile platform to use as a base station depends on two major data profile requirements namely real time, defining network abilities of the base station such as cellular network support in case of emergency transmissions for example, while data processing could define the power of computing needed to be performed at base station if local processing is required. BS as a gateway should handle interoperability between different communication technologies to relay between intra and beyond-BAN segments.

- 4) Communication between Sensors and BS. Low energy short range communications should be used to communicate with sensors such as ANT+, IEEE 802.15.1 low energy (LE) ${ }^{1}$, IEEE 802.15.4, ISM band or UWB. Among theses, IEEE 802.15.4 LE seems to be a proper choice especially with the use of a Smartphone base station. Compared to IEEE 802.15.4 (Zigbee), IEEE 802.15.1 LE is in fact more and more built-in in Smartphones bringing a number of

${ }^{1}$ BLE for Bluetooth Low Energy or more recently Bluetooth Smart 
advantages going in favor of mobility support as described in [21] and [22]. In home environment, if BS is implicated in the upload of patients data, communication with sensors can be either performed directly such as in outdoor environment or through low energy communicating relays, when the patient puts its BS far from him at home for example; relays can be represented by a fixed ambient sensor network (see next recommendation).

- 5) Ambient sensor network (ASN). Optional and sometimes mandatory depending on the target application and its medical requirements. Although sensors have typically a continuous power source, using low energy technology such IEEE 802.15.1 LE is also recommended for compatibility reasons with mobile battery-dependant devices. ASN might also need to support multi-hop communications, as well as larger range communication technology such as 802.11 either on each node or on the nearest node to the gateway, in case the ASN is used as a relay network for the BS or the sensors to the gateway. Even if some low energy technologies such as 802.15.1 LE were basically designed to be used in single hop star topologies, it is however possible to design mesh topologies as mentioned before as well as it was reported in [23] and [24]. The need or the selection of parameters related to an ASN might be dependant of data correlation requirement defining for example if some data from body sensors need to be augmented using data from ambient sensors in order to deduce patient's relevant health information.

- 6) Intra-WBAN topology. Single-hop (star) and multihop (mesh) topologies can be both used for intraWBAN. Although single-hop strategy could be basically sufficient considering the supported range and human body dimensions, multi-hop strategy, even if more complex in realization, might be however necessary for reliability reasons especially in outdoor settings [7], moreover, it was reported that multi-hop in addition to possibly reducing diffraction-caused path losses, contributes in the well-distribution of power consumption among sensors [25], as it was done for the energy-efficient routing protocol in [26]; a comparison between single and multi hop topologies can be consulted in [25]. For this, adaptation of short range communication technology for such situations as previously seen might need the implementation of additional protocols through advanced features, as it was done for 802.15.1 LE in [27] for example. The selection of appropriate intra-WBAN topology is therefore in close relation with data reliability requirement. Furthermore, real-time as well as data prioritization requirements are also important for the selection, such as for example a failure of a single sensor that might perturbate a whole set of other sensors possibly requiring real-time collection [25].

\section{Inter-BAN level}

- 7) In-home gateway and relays. In home environment, the gateway can be any communicating device connected to the WAN (Internet), such as typically a home PC, a laptop or a wireless router. It should of course support the right communication technologies to be able to play its role of gateway on the beyond-BAN.
If the gateway device supports only short range connectivity such as $802.15 .1 \mathrm{LE}$, an infrastructure of relays covering the house such as the ASN should be used in order to relay data from sensors / BS to the gateway.

- 8) Cloudlets. Considered as small scale cloud systems bringing closer cloud capabilities to mobile devices; these are interesting for the support of mobility in WHMS. In fact, deployment of cloudlets in users' environment (especially outdoor), in addition to support network scalability, could reduce transmissions cost and energy if these are used as relays, through lower power technologies such as 802.11 comparing to direct communications via costly technologies such as 3G or LTE [45]. Cloudlets should support suitable communication technology used by BS such as 802.11 and be connected with Remote side through wide communication technology such as 802.16 or ideally through wired communication for better reliability.

- 9) Communication between BS and Remote side (RS). In outdoor environment, the base station should be able to offer different possibilities of connectivity depending on the available networks. It should be able to select the best network to use (Wi-Fi hotspots, cellular ...) depending on different parameters using a mechanism known as handover (see next section "key mechanisms"). This network selection is dependent on data profile constraints in term of real-time, data reliability and data management requirements. However, selecting cloudlet network if available can be prioritized because of its advantageous capabilities i.e. storage and processing power for faster advanced health analysis, as well as cost and energy effective and eventually more reliable communication. In an in-home environment, the communication is generally performed through the in-home gateway as it will be described in the next point. However, in some situations such as emergency cases or WAN connection lost, the communication can be done directly between BS and RS through cellular networks, which makes the selection dependant on real-time and data prioritization requirements.

- 10) Communication through in-home gateway. The transmission of data to the remote side typically via Internet can be done either directly or via the base station if this latter is implicated. However, avoiding $\mathrm{BS}$ implication is not recommended since BS is very useful for local processing and emergency transmission through cellular networks. BS implication depend therefore mainly on real-time, data processing as well as data correlation requirements of data profile. BS or Sensors communicate with in-home gateway through available interfaces on both sides such as 802.15.1 LE. If needed such as in case of short range technology, communication can be done through relays which should further support both BS/Sensors and in-home gateway interfaces if these are different. ASN can play the role of the relay infrastructure.

\section{Beyond-BAN level}

- 11) Remote side. Can be represented by a medical private cloud offering needed resources in term of storage, computing and communication capabilities. Internet connectivity is needed in order to share 
different patient-related information with family and health professionals. This can be done through web service-based architecture $[2,10]$ and accessed via web or mobile applications. Mobile platforms are worth to be used by health professionals as well as families for continuous monitoring of their patients anywhere and anytime including advising them and managing sensing and system parameters such as thresholds [18,28,29]. Web services to implement at remote side are closely dependant from data management requirement of data profile.

\section{Key mechanisms}

One of the main concerns when supporting mobility in HMS is maintaining connectivity and quality of service (QoS) at a sufficient level meeting application requirements $[12,29,41]$. Furthermore, WHMS relying on wireless technologies, energy as well as cost are important criteria to be considered, where from one hand, the more energy consumption is optimized the more the monitoring sustainability and convenience of the user increase, while from another hand, cost-effectiveness allows such systems to be more affordable and accessible $[12,40]$. Besides, because of surrounding environment changing of a mobile user such as in term of network coverage, context awareness should be considered $[31,41]$.

In order to cope with these concerns, a number of means supporting context awareness should be designed and implemented within WHMS. A set of key mechanisms for supporting mobility are briefly described and presented below in the form of design requirements, along with associated data profile requirements.

It is noted that basically different algorithms used in following mechanisms should be designed and implemented to be energy efficient and not constitute or leads to any kind of security vulnerabilities.

1) Handover. Context-awareness decisional mechanism dealing with network coverage change by switching between points of attachment (PA). Handovers allow to maintain connectivity, maximize throughput and support QoS [12]. We talk about micro/macro mobility handovers whether the switching is between the same network domain PAs (keeping same IP address) or through different network domains PAs [29]; Horizontal/vertical handovers from another hand distinguish between transitions through homogeneous or heterogeneous network technologies [38]. Handover decisions are performed on the basis of a number of parameters such as RSSI (Received Signal Strength Indication) [12,30,31]. Power consumption and cost are important parameters to be taken in consideration by handover decision algorithms and a tradeoff between quality of the connections and others parameters such as cost might be considered [32,41]. Handover decision algorithm should be designed to perform handovers in a seamless manner, avoiding unnecessary handovers while being energy efficient [31]. Mechanisms for data buffering should also be designed in order to locally store temporary data in case for example of a slow handover. Required handover time of performing is highly related to the data profile real-time and frequency requirements. In some cases, a handover can be not necessary, even if the current connection is lost such as in case of very small data collection frequency like one measurement per day, or in case of delay tolerance like in applications where collected data are transmitted to remote side after a number of days. However, these cases are not emergencies aware since the connectivity is not maintained as much as possible.

2) Intermittent connectivity handling. Handling intermittent connectivity is typically performed through switching to DTN (Delay-Tolerant Networking) communication mode that handles temporary ruptures of connectivity [20] which might be more necessary in rural environments. DTN "Store and forward" algorithms require local storage capability as well as possibly local computing ability and a minimum of processing algorithms for real-time analysis and continuous emergencies detection. Moreover, because of the connectivity ruptures and delayed transmissions, remote-side algorithms for processing-out received data are needed including ordering and synchronization [18]. DTN communication mode features are closely related to data profile real-time requirement defining for example if local processing is needed or not, data prioritization requirement is also important to differentiate between data that could support delay and those that don't whether it is for processing or uploading, as well as prioritizing data handling on reconnection. Lastly, data frequency requirement could determine whether DTN mode should be used or not. From another hand, intermittent connectivity might be mitigated through wireless coverage extension by increasing network connectivity if node cooperation with its neighboring is possible [43].

3) Coexisting of Networks. A monitored user can be surrounded by different wireless technologies. Its body network therefore coexists with this environment whether it comes to other surrounding body networks, called homogeneous coexistence, or other wireless networks around within the same range called heterogeneous coexistence [13]. Obviously, this coexistence causes interferences resulting in the loss of monitored user's health data and other traffic; thus, coexistence handling requirement is closely dependent from reliability needs, knowing that many applications require high reliability particularly in emergency situations [21]. It is reported that up to now, current standards haven't yet properly address coexistence problem between WBAN and other sameband surrounding networks [21]. Mechanisms to detect [33] as well as to mitigate [34] coexistence interferences impact are therefore necessary.

4) Localization and tracking. Mechanisms for localization are of high importance, as a user being mobile especially elderly should always be trackable in real-time so that if an emergency is detected, the user can be immediately located and found [29]. Since a mobile user roams between indoor and outdoor environments where mechanisms for localization should be location-context aware and thus differs, algorithms to differentiate between in-home and outdoor should be used which might further allow to take advantage of the user's location. In in-home environment, localization algorithms could rely on different fixed home wireless infrastructures such as the ASN if available; Depending on target application requirements, Wireless Multimedia Sensor Networks (WMSN) might also be used as 


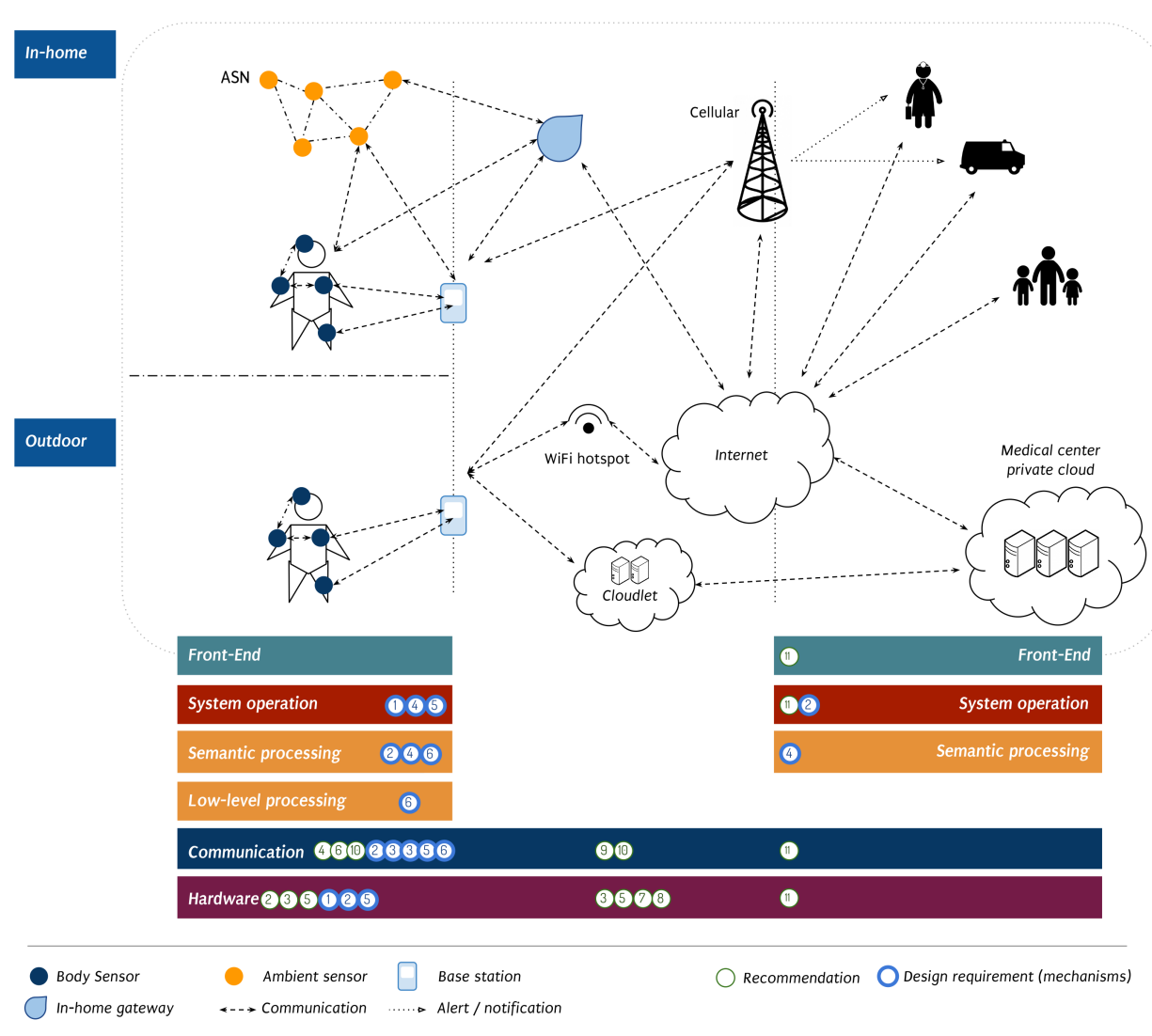

Fig. 2 Illustration of design framework for mobility support in WHMS

they are reported to provide more information than classical WSN but need however energy-aware algorithms [42]. Regarding outdoor, mobile platforms used as base station especially Smartphones are typically equipped with GPS sensor on which localization algorithms can easily rely; information provided by cellular networks through Location Area Identity (LAI) can also be used in order to calculate user position [35].

5) Cost-effective and energy-saving Transmission schemes. For a system that supports mobility, saving energy and mitigating communication costs are important; for this, scheme of transmission used should be context aware and flexible so that it can adapt to different situations on the basis of application requirements. If for example, in a given application, some of the data historization at remote side is not used while their processing is not complex, this latter can be performed at the base station and only emergency data or alerts are transmitted such as in [2], whereas for the rest of data, a periodic transmission can still be performed. Another examples are to proceed to the transmission only if values changed, or also to differentiate between in-home and outdoor communications in the way that in in-home where remote connectivity can be costless, all data can be transmitted periodically while in outdoor where transmission's cost is more important, thresholds can be for example used and only data exceeding this latter are sent $[26,35]$; or, only data that might need a remote processing for computing power needs for example are transmitted. Appropriate transmission scheme would therefore depend on data processing, prioritization and real-time requirements. Algorithms for managing the schema of transmission are therefore also needed. These are typically based on a given criteria such as threshold, data classification into emergency/routine or network infrastructure used, and might require local storage and computing capabilities. The overall mechanism algorithms should be able to classify given criteria information such as differentiating between routine and emergency data and messages, then take relevant decision on the basis of this classification.

6) Intra-WBAN mechanisms. In order to handle intraWBAN mobility (i.e. body movements) interferences, algorithm-based methods should be used to mitigate interferences impact [25]. These methods can be either implemented at sensors-level if supported or at BS-level where techniques can be more advanced due to the power of base station compared to sensors. Such algorithms are typically dependant of data reliability requirement constraints as the more reliability is required; the more the interferences should be mitigated. From another hand, as previously seen in the recommendations, if a multi-hop strategy is used, a mechanism for its support is required if not built-in such as in case of BLE, as well as a mechanism for switching between the single and multi-hop topologies.

\section{E. Framework's discussion}

Mobility support involves different design aspects of WHMS. Design requirements previously defined can be spotted on design layers in order to draw a global idea of the level of implication of each aspect (Fig. 2).

On the front-end layer, mobile and web applications used by doctors and family should be designed with ergonomic and easy to use interfaces. 
System operation layer comprises within intra-WBAN, algorithms used in mobility support mechanisms including handover, localization and transmission schemes, while within beyond-WBAN algorithms for processing-out data are needed for intermittent connectivity handling as well as various algorithms for remote access such as authentication.

Regarding semantic processing layer, this comprises important algorithms for localization, intermittent connectivity handling and movements' interferences mitigation mechanisms.

Low-level processing layer might also involve algorithms for movements' interferences mitigation mechanisms.

Besides, communication and physical layers are the most involved design aspects for mobility support.

Communication layer comprises within intra-WBAN sensors and base station topology, communication standards between sensors and BS as well as between sensors and in-home gateway, and a set of algorithms for mechanisms including handover, coexistence handling, transmission schemes and single/multi-hop switching. Within inter-WBAN, are covered communication standards and networks used between base station and remote side as well as with in-home gateway and between this latter and remote side. Internet connectivity is required and web service architecture is recommended at beyond-WBAN level.

Regarding physical layer, and within intra-WBAN, recommendations around sensors design such as energy efficiency and solidity should be defined; while the base station hardware would be a mobile platform preferably a Smartphone and an optional ambient sensor network could be deployed for augmenting body sensors data as well as relaying them; cloudlets from another hand can also be deployed as intermediate small scale clouds and reliable relays saving cost and energy regarding BS communication. Moreover, handover, intermittent connectivity handling and transmission schemes mechanisms might require some specific needs regarding local storage and computing. Within inter-WBAN, base station, in-home gateway and ambient sensor network interfaces characteristics are defined. Lastly, within beyondWBAN, mobile platforms are recommended for health professionals and family in order to follow patients anywhere and anytime.

\section{CONCLUSION AND FUTURE WORK}

In this paper, we proposed a design framework taking in consideration patient context constraints with the aim to serve as a starting point for the conception and possibly the evaluation of health monitoring systems with mobility support. The framework was schematized by double projecting pointed out design requirements for key mechanisms, and a number of conceptual recommendations, on the three-tiers representation, as well as the introduced design layers. From the design layers of the framework, it was noted that communication and physical aspects were the most involved aspects for mobility support; this can explained by the fact that mobility essentially affects transmissions between patients sensors and remote center which require appropriate communication protocols, standards and so on, while the mobility freedom of the patient requires equipments with specific characteristics as it was described in this work. For future perspectives, presented design framework should be practically validated through simulations and experimentations where mechanisms should be designed and implemented within targeted application and context, as well as different conceptual recommendations where available technologies could be compared in order to validate their suitability.

\section{References}

[1] A. Pantelopoulos and N. Bourbakis, 'A Survey on Wearable SensorBased Systems for Health Monitoring and Prognosis', IEEE Transactions on Systems, Man, and Cybernetics, Part C (Applications and Reviews), vol. 40, no. 1, pp. 1-12, 2010.

[2] C. Chen, A. Knoll, H. Wichmann and A. Horsch, 'A review of threelayer wireless body sensor network systems in healthcare for continuous monitoring', Journal of Modern Internet of Things, vol 2, iss 3, 2013.

[3] M. Fouad, N. El-Bendary, R. Ramadan and A. Hassanien, 'Wireless Sensor Networks: A Medical Perspective', in Wireless Sensor Networks: Theory and Applications, USA, CRC Press, Taylor and Francis Group, pp. 713-732, 2013

[4] F. Miao, 'MobiHealthcare System: Body Sensor Network Based MHealth System for Healthcare Application', ETSN, vol. 01, no. 01, pp. 12-18, 2012.

[5] F. Miao, 'MobiHealthcare System: Body Sensor Network Based MHealth System for Healthcare Application', ETSN, vol. 01, no. 01, pp. 12-18, 2012.

[6] M. Al-Bashayreh, N. Hashim and O. Khorma, 'A survey on success factors to design application frameworks to develop mobile patient monitoring systems', 2012 IEEE EMBS Conference on Biomedical Engineering and Sciences (IECBES) 17-19 Dec, pp. 57-62, 2012

[7] S. Patel, H. Park, P. Bonato, L. Chan and M. Rodgers, 'A review of wearable sensors and systems with application in rehabilitation', Journal of neuroengineering and rehabilitation, vol 9, iss 1, p. 21, 2012

[8] A. Hadjidj, M. Souil, A. Bouabdallah, Y. Challal and H. Owen, 'Wireless sensor networks for rehabilitation applications: Challenges and opportunities', Journal of Network and Computer Applications, vol 36, iss 1, pp. 1-15, 2013

[9] T. Ahmed, K. Kyamakya and M. Ludwig, 'Architecture of a contextaware vertical handover decision model and its performance analysis for GPRS-WiFi handover', 11th IEEE Symposium on Computers and Communications, 2006. ISCC '06. Proceedings, pp. 795-801, 2006.

[10] K. Elgazzar, M. Aboelfotoh, P. Martin and H. Hassanein, 'Ubiquitous Health Monitoring Using Mobile Web Services', Procedia Computer Science, vol. 10, pp. 332-339, 2012.

[11] E. Egbogah and A. Fapojuwo, 'A survey of system architecture requirements for health care-based wireless sensor networks', Sensors, vol 11, iss 5, pp. 4875-4898, 2011.

[12] P. Khan, N. Ullah, S. Ullah and K. Kwak, 'Seamless Interworking Architecture for WBAN in Heterogeneous Wireless Networks with QoS Guarantees', J Med Syst, vol. 35, no. 5, pp. 1313-1321, 2011.

[13] M. Deylami and E. Jovanov, 'A Distributed Scheme to Manage The Dynamic Coexistence of IEEE 802.15.4-Based Health-Monitoring WBANs', IEEE J. Biomed. Health Inform., vol. 18, no. 1, pp. 327-334, 2014.

[14] D. Yang, Y. Xu, and M. Gidlund, 'Wireless coexistence between IEEE 802.11- and IEEE 802.15.4-based networks: A Survey', Int. J. Distrib. Sensor Netw., vol. 2011, pp. 1-17, 2011.

[15] K. Bian, J. Park and B. Gao, 'Cognitive Radio Networks: Medium Access Control for Coexistence of Wireless Systems’, Springer 2014.

[16] H. Liu, H. Darabi, P. Banerjee and J. Liu, 'Survey of Wireless Indoor Positioning Techniques and Systems', IEEE Trans. Syst., Man, Cybern. $C$, vol. 37, no. 6, pp. 1067-1080, 2007.

[17] U. Anliker, J. Ward, P. Lukowicz, G. Troster, F. Dolveck, M. Baer, F. Keita, E. Schenker, F. Catarsi, L. Coluccini and others, 'AMON: a wearable multiparameter medical monitoring and alert system', IEEE Transactions on Information Technology in Biomedicine, vol 8, iss 4, pp. 415-427, 2004.

[18] P. Kulkarni and Y. Ozturk, 'Requirements and design spaces of mobile medical care', ACM SIGMOBILE Mobile Computing and Communications Review, vol 11, iss 3, pp. 12-30, 2007.

[19] Z. Sanaei, S. Abolfazli, A. Gani and R. Buyya, 'Heterogeneity in Mobile Cloud computing: Taxonomy and Open Challenges', IEEE Commun. Surv. Tutorials, vol. 16, no. 1, pp. 369-392, 2014.

[20] D. Benferhat, 'Conception d'un système de communication tolérant la connectivité intermittente pour capteurs mobiles biométriques Application à la supervision médicale de l'activité cardiaque de marathoniens', 2013. 
[21] R. Cavallari, F. Martelli, R. Rosini, C. Buratti and R. Verdone, 'A Survey on Wireless Body Area Networks Technologies and Design Challenges', IEEE Communications Surveys \& Tutorials, vol. 16, no. 3, 3rd quarter 2014.

[22] http://www.bluetooth.com/Pages/Press-Releases-Detail.aspx?ItemID=4 Accessed 14-02-2015

[23] http://www.marketwatch.com/story/samsung-unveils-industry-firstbluetooth-mesh-network-enabled-smart-led-light-bulb-2014-06-03 Accessed: 12/02/2015

[24] http://www.csr.com/news/pr/2014/csr-mesh - Accessed: 12/02/2015

[25] X. Lai, Q. Liu, X. Wei, W. Wang, G. Zhou and G. Han, 'A survey of body sensor networks', Sensors, vol 13, iss 5, pp. 5406-5447, 2013.

[26] S. Yousaf, M. Akbar, N. Javaid, A. Iqba1, Z. A. Khan, U. Qasim, 'CEMob Critical Data Transmission in Emergency with Mobility Support in WBANs', 2014 IEEE 28th International Conference on Advanced Information Networking and Applications.

[27] K. Mikhaylov and J. Tervonen, 'Multihop Data Transfer Service for Bluetooth Low Energy', 2013 13th International Conference on ITS Telecommunications (ITST).

[28] P. J Fortier, B. Viall, 'Development of a Mobile Cardiac Wellness Application and Integrated Wearable Sensor Suite', 2011 5th International Conference on Sensor Technologies and Applications (SENSORCOMM).

[29] Z. Lv, F. Xia, G. Wu, L. Yao, Z. Chen, 'iCare: A Mobile Health Monitoring System for the Elderly', 2010 IEEE/ACM International Conference on Green Computing and Communications \& 2010 IEEE/ACM International Conference on Cyber, Physical and Social Computing.

[30] J. Caldeira, J. Rodrigues and P. Lorenz, 'Toward ubiquitous mobility solutions for body sensor networks on healthcare', IEEE Communications Magazine, vol. 50, no. 5, pp. 108-115, 2012.

[31] J. Caldeira, J. J. Rodrigues and P. Lorenz, 'Intra-Mobility Support Solutions for Healthcare Wireless Sensor Networks-Handover Issues', IEEE Sensors Journal, vol. 13, no. 11, November 2013.

[32] J. Silva, A. Arsenio and N. Garcia, 'Context-awareness for mobility management: A systems survey for healthcare monitoring', 2011 6th International Conference on Broadband and Biomedical Communications (IB2Com), pp. 18-23, 2011.

[33] Y. Han, Z. Jin, J. Cho, and T.S. Kim, 'A Prediction Algorithm for Coexistence Problem in Multiple WBANs Environment', 2014 International Conference on Ubiquitous Information Management and Communication ICUIMC (IMCOM).
[34] C.F. Chiasserini and R.R. Rao, 'Coexistence Mechanisms for Interference Mitigation in the 2.4-GHz ISM Band', IEEE Tansactions on Wireless Communications, vol. 2, no. 5, September 2003

[35] A. Bourouis, M. Feham and A. Bouchachia, 'A New Architecture of a Ubiquitous Health Monitoring System A Prototype of Cloud Mobile Health Monitoring System', IJCSI International Journal of Computer Science Issues, Vol. 9, Issue 2, No 2, March 2012

[36] D. Barakah and M. Ammad-uddin, 'A survey of challenges and applications of wireless body area network (WBAN) and role of a virtual doctor server in existing architecture', 2012 Third International Conference on Intelligent Systems, Modelling and Simulation (ISMS), p.214-219, 2012.

[37] M. Baig, H. Gholamhosseini and M. Connolly, 'A comprehensive survey of wearable and wireless ECG monitoring systems for older adults', Medical \& Biological Engineering \& Computing, vol. 51, no. 5, pp. 485495, 2013.

[38] R. Bolla and M. Repetto, 'A Comprehensive Tutorial for Mobility Management in Data Networks', IEEE Commun. Surv. Tutorials, vol. 16, no. 2, pp. 812-833, 2014.

[39] Sana Ullah, Muhammad Imran, and Mohammed Alnuem, 'A Hybrid and Secure Priority-Guaranteed MAC Protocol for Wireless Body Area Network', International Journal of Distributed Sensor Networks, vol. 2014.

[40] Hamie, J.; Denis, B.; Richard, C., 'Constrained decentralized algorithm for the relative localization of wearable wireless sensor nodes', Sensors, 2012 IEEE , pp.1,4, 28-31 Oct. 2012.

[41] Cherkaoui, E.H.; Agoulmine, N., 'Context-aware mobility management with WiFi/3G offloading for ehealth WBANs', e-Health Networking, Applications and Services (Healthcom), 2014 IEEE 16th International Conference on , vol., no., pp.472,476, 15-18 Oct. 2014.

[42] Boulanouar, I.; Rachedi, A.; Lohier, S.; Roussel, G., 'Energy-aware object tracking algorithm using heterogeneous wireless sensor networks', Wireless Days (WD), 2011 IFIP , vol., no., pp.1,6, 10-12 Oct. 2011.

[43] Gueguen, C.; Rachedi, A.; Guizani, M., 'Incentive Scheduler Algorithm for Cooperation and Coverage Extension in Wireless Networks', Vehicular Technology, IEEE Transactions on , vol.62, no.2, pp.797,808, Feb. 2013.

[44] P. Pawar, V. Jones, B. Van Beijnum and H. Hermens, 'A framework for the comparison of mobile patient monitoring systems', Journal of biomedical informatics, vol 45, iss 3, pp. 544-556, 2012.

[45] M. Quwaider and Y. Jararweh, 'Cloudlet-based Efficient Data Collection in Wireless Body Area Networks', Simulation Modelling Practice and Theory, vol. 50, pp. 57-71, 2015. 